\title{
Antizyme inhibitor 1 genetic polymorphisms associated with diabetic patients validated in the livers of diabetic mice
}

\author{
CHENG-HSU CHEN $^{1-4^{*}}$, YEH-HAN WANG ${ }^{5^{*}}$, SHANG-FENG TSAI $^{2,3,6}$, \\ TUNG-MIN YU ${ }^{2,4}$, SHIH-YIN CHEN ${ }^{7,8}$ and FUU-JEN TSAI ${ }^{7-9}$
}

\begin{abstract}
${ }^{1}$ Department of Medical Research, Division of Basic Medical Sciences; ${ }^{2}$ Department of Internal Medicine, Division of Nephrology, Taichung Veterans General Hospital; ${ }^{3}$ Department of Life Science, Tunghai University, Taichung 40705; ${ }^{4}$ School of Medicine, China Medical University, Taichung 40402;

${ }^{5}$ Department of Anatomical Pathology, Taipei Institute of Pathology, School of Medicine, National Yang-Ming University;

${ }^{6}$ Department of Internal Medicine, School of Medicine, National Yang-Ming University, 11221 Taipei; ${ }^{7}$ School of Chinese Medicine, China Medical University, Taichung 40402; ${ }^{8}$ Genetics Center, Medical Research; 9 Department of Medical Genetics, China Medical University Hospital, Taichung 40447, Taiwan, R.O.C.
\end{abstract}

Received October 31, 2018; Accepted June 6, 2019

DOI: $10.3892 /$ etm.2019.7919

\begin{abstract}
Diabetes mellitus (DM) is a complex disease caused by absolute or relative insulin deficiency. The C57BLKsJ-db/db mouse model is a useful animal model for studying type 2 DM (T2DM). The present study investigated the association between an antizyme inhibitor 1 (AZIN1) gene polymorphism (rs1062048) and T2DM susceptibility in 2,270 Taiwanese individuals (570 patients with T2DM and 1,700 controls). Additionally, the present study investigated AZIN1 gene and protein expression in the liver tissues of mice in three age groups (4, 16 and 32 weeks) through reverse transcription-quantitative PCR, western blotting and immunohistochemistry. The data indicated that the genotype frequency distribution of the rs1062048 single-nucleotide polymorphism differed significantly between the patients with T2DM and controls $(\mathrm{P}<0.05)$. Furthermore, gene and protein expression levels of AZIN1 were significantly lower in early stage and late stage T2DM mouse liver samples than in control samples. Overall, the data suggested that AZIN1 expression is involved in T2DM development.
\end{abstract}

Correspondence to: Dr Shih-Yin Chen or Dr Fuu-Jen Tsai, Genetics Center, Medical Research, China Medical University Hospital, 2 Yuh-Der Road, Taichung 40447, Taiwan, R.O.C.

E-mail: chenshihy@gmail.com

E-mail: d0704@mail.cmuh.org.tw

${ }^{*}$ Contributed equally

Key words: C57BLKsJ-db/db mouse, type 2 diabetes mellitus, antizyme inhibitor 1

\section{Introduction}

Globally, the number of adults affected by diabetes mellitus (DM) increased rapidly from $\sim 108,000,000$ in 1980 to $422,000,000$ in 2014 (1). In the adult population, the global prevalence of DM has nearly doubled from 4.7 to $8.5 \%$ since 1980 (1). The prevalence of DM in adults reported by the International Diabetes Federation Western Pacific Region ranges between $2.6 \%$ in Cambodia and $16.9 \%$ in Malaysia (2). The major risk factors for type 2 DM (T2DM) are age, metabolic conditions (overweight and obesity) and lifestyle (unhealthy diet, physical inactivity and smoking); however, genetic factors, including ethnicity, family history of diabetes and previous gestational diabetes may serve crucial roles in increasing T2DM susceptibility (1). The mean height, weight, body mass index (BMI) and total visceral fat volume are lower in the East Asian population compared with in the African and Caucasian populations; however, non-obese East Asian individuals can also develop T2DM (3-6). A family history of T2DM is a crucial factor. If a parent has T2DM, their children are $40 \%$ as likely to develop T2DM. If both parents have T2DM, the probability of their children developing T2DM exceeds $70 \%(7,8)$. In addition, different ethnic groups exhibit different incidence rates; this also demonstrates the effects of genetic factors (9). In the majority of individuals, T2DM causes insulin resistance in its target tissues throughout the body, and eventually the b-cells of the pancreatic islets fail to secrete sufficient insulin to overcome the resistance. In addition to environmental factors, multiple genetic factors appear to cause predisposition to T2DM $(10,11)$. Currently, $>120$ associated gene variants have been identified and have minor effects on diabetes risk (12). There is no complete information regarding genetic susceptibility of T2DM despite the availability of novel detection techniques; thus, additional pieces of the puzzle may help to explain the heritability of diabetes.

Polyamines are organic polycations essential for cell growth and differentiation, and impairment of the polyamine signaling 


\section{Homo sapiens chromosome 8}

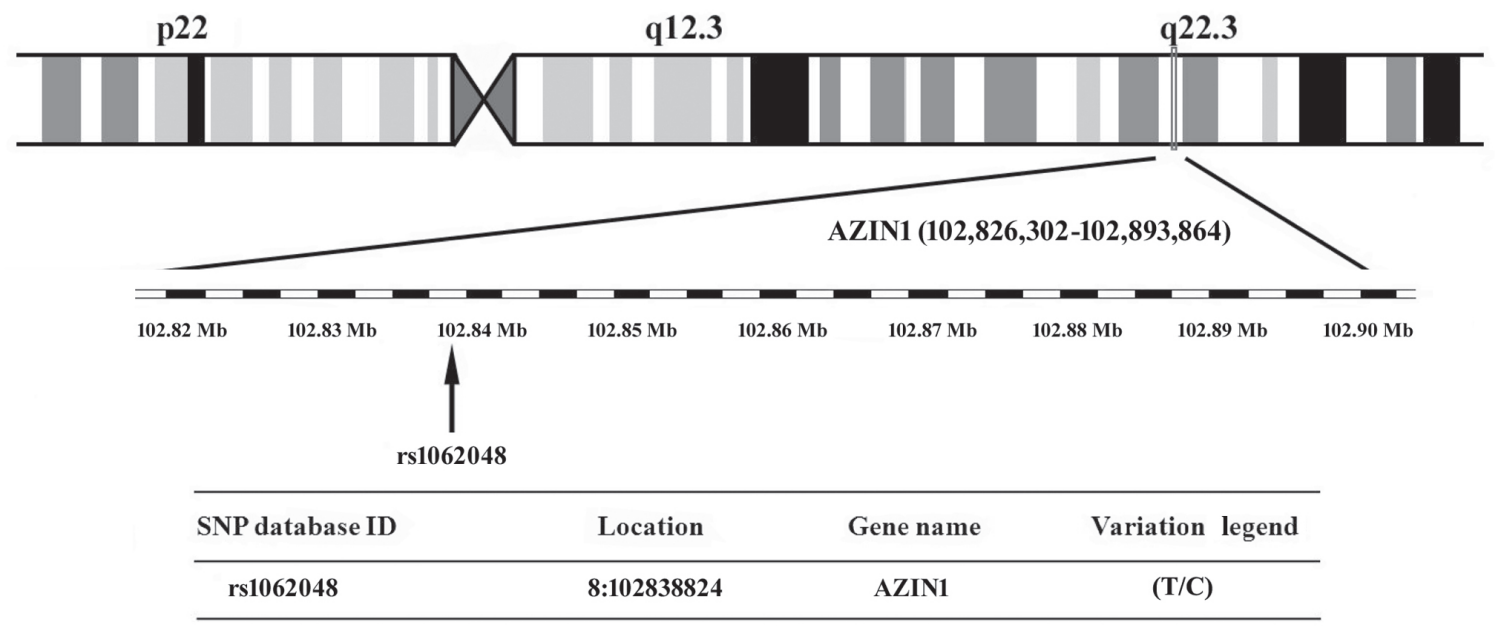

Figure 1. Map of AZIN1 (rs1062048) located within chromosome 8q22.3 region (102,826,302-102,893,864 bp). AZIN1, antizyme inhibitor 1.

pathway can lead to numerous diseases and conditions, including cancer, inflammation, stroke, renal failure and diabetes (13). Ornithine decarboxylase (ODC) is involved in the commitment and rate-limiting step in polyamine biosynthesis, and polyamine homeostasis is maintained by the regulatory proteins antizyme isoform 1 (AZ1) and antizyme inhibitor 1 (AZIN1) (14). Translation of intracellular AZ1 occurs alongside upregulation of intracellular polyamine synthesis $(15,16)$. AZ1 binds to ODC to form an ODC-AZ1 heterodimer that inhibits polyamine production (14). AZIN1 is obtained from rat liver extract, exhibits high similarity in sequence and structure to ODC, and has a greater affinity for AZ1 than ODC (17). AZIN1 inhibits the activity of ODC, promotes ubiquitin-dependent degradation of ODC and contributes to carcinogenesis (17). However, binding of AZ1 to ODC reduces the cellular polyamine level and affects the conversion of ASPC- 1 cells into $\alpha$-cells, which form islet-like structures and express the glucagon gene to regulate pancreatic endocrine cell function (18). Competition between AZIN1 and ODC for AZ1 binding effectively restores ODC activity and increases the cellular polyamine level, which may serve a role in diabetes (16). The present study demonstrated that AZIN1 genetic polymorphisms are potential candidate genes for diabetes in the Taiwanese population and revealed the downregulation of AZIN1 gene expression in T2DM.

\section{Materials and methods}

Patients and sample collection for genotyping. In the present study, a total of 570 T2DM patients, aged 20 years and older (mean age, 63.6 $\pm 11.5 ; 51.4 \%$ male individuals) were enrolled at China Medical University Hospital (Taichung, Taiwan) between August 2014 and July 2015. All patients met the diagnostic criteria (19) for T2DM with the exclusion criteria those without T2DM. To determine the prevalence of polymorphism in these patients, genotype frequency data of 1,700 healthy controls was downloaded from the Taiwan Biobank (https://taiwanview.twbiobank.org.tw/) (case no: TWBR10509-02; control no: TWBR10309-001) (9). In addition, to determine the association between polymorphism and clinical features in T2DM patients, the clinical features, including age, BMI, hemoglobin A1c, blood urea nitrogen, creatinine, uric acid, total calcium, phosphorus, parathyroid hormone (PTH), albumin, cholesterol, triglycerides, low-density lipoprotein-C and alanine aminotransferase (ALT) were also downloaded from the Taiwan Biobank. The present study obtained the rs1062048 single-nucleotide polymorphism (SNP) at chromosome region 8q22.3 in AZIN1 (Fig. 1) from the National Center for Biotechnology Information's SNP database (http://www.ncbi.nlm.nih.gov/snp). The SNPs in the T2DM and control groups were then compared. Tag SNPs were selected using the Tagger function (http://software. broadinstitute.org/mpg/tagger/server.html) with the additional criteria: ii) A threshold minor allele frequency in the HapMap phase 3; and ii) Han Chinese in Beijing, China (CHB) + Japanese in Tokyo, Japan (JPT) population of 0.05 for 'tag SNPs'. Chi-square tests were used to calculate odds ratios and P-values. All study protocols were approved by the Ethical Committee of China Medical University Hospital (approval no. CMUH103-REC2-071). Informed consent was obtained from all analyzed patients.

Animal model. A total 24 male background BKS.Cg-Dock $7^{\mathrm{m}}+/+$ Lepr $^{\mathrm{db}} / \mathrm{JNarl}$ mice (age, 4 weeks old) were purchased from the National Laboratory Animal Center. Mice with the + Dock $7^{\mathrm{m}} /+$ Dock $7^{\mathrm{m}}$ genotype constituted the control group $(n=12)$ and those with the $+\operatorname{Lepr}^{\mathrm{db}} /+\operatorname{Lepr}^{\mathrm{db}}$ genotype constituted the T2DM group $(n=12)$. The diabetic mice averaged $62 \pm 3 \mathrm{~g}$ in body weight at the time of the studies and their control littermates averaged $20 \pm 1 \mathrm{~g}$. The animals were housed in individual cages and provided with lab chow ad libitum (Lab Diet $5 \mathrm{k} 52$; Purina) in a room at $22-25^{\circ} \mathrm{C}$, with sufficient water, a relative humidity of $50-70 \%$, and a 12 -h light/dark cycle. The present study involved animal experiments and considered the 3R principles of 'Replace', 'Reduce' and 'Refine' to optimize experimental design (20). According to the information from Jackson Laboratory (JAX stock \#000642) (21), the mouse model exhibited elevated blood sugar at 4-8 weeks and mortality by 10 months of age. The mice in the present study 
were divided into six groups (four mice per group), namely three control groups of mice aged 4 (early stage), 16 (middle stage) and 32 (late stage) weeks, and three T2DM groups of mice aged 4 (early stage), 16 (middle stage) and 32 (late stage) weeks. Mice were sacrificed and the liver tissue was obtained at the scheduled time. Sample went through the methodology of flash freezing (22), which provided excellent specimen integrity and a wide array of options for tissue analysis, including extraction of RNA and proteins in the present research. The present study was reviewed and approved by the Institutional Animal Care and Use Committee (IACUC) of China Medical University (IACUC permit no. 2106-221).

Reverse transcription-quantitative PCR (RT-qPCR). The RNeasy Mini kit (Qiagen, Inc.) was used for total RNA isolation from the liver tissues of the control and T2DM mice, and the Superscript First-Strand Synthesis kit (Invitrogen; Thermo Fisher Scientific, Inc.) was used for complementary DNA transcription, as previously described (23). Briefly, the cDNA synthesis reaction protocol including reverse transcription step, $30 \mathrm{~min}$ at $42^{\circ} \mathrm{C}$ and $\mathrm{RT}$ inactivation step, $1 \mathrm{~min}$ at $95^{\circ} \mathrm{C}$. To study gene expression, qPCR was performed using the ABI ViiA ${ }^{\mathrm{TM}} 7$ Real-Time PCR system (Applied Biosystems; Thermo Fisher Scientific, Inc.) and TaqMan ${ }^{\mathrm{TM}}$ MGB (minor groove binder)-NFQ (nonfluorescent quencher) Universal ProbeLibrary (Roche Diagnostics) probes, as previously described (24). The primer sequences were as follows: Mus musculus AZIN1 (NM_018745.5) forward, 5'-TGCTAAGAA AGTTGTTGAAAATGATAA-3'; murine AZIN1 reverse, 5'-CTGGCTCATCACTCCCATTT-3' with UPL probe \#6 (Roche, cat. no. 04685032001); mouse GAPDH (M32599.1) forward, 5'-GAGCCAAACGGGTCATCA-3'; and murine GAPDH reverse, 5'-CATATTTCTCGTGGTTCACACC-3' with UPL probe \#29 (Roche, cat. no. 04687612001). PCR amplification conditions were: Initial denaturation at $95^{\circ} \mathrm{C}$ for $5 \mathrm{~min}$, followed by 25 cycles of $95^{\circ} \mathrm{C}$ for $10 \mathrm{sec}, 56^{\circ} \mathrm{C}$ for $10 \mathrm{sec}$ and $72^{\circ} \mathrm{C}$ for $20 \mathrm{sec}$, with a final extension at $72^{\circ} \mathrm{C}$ for $5 \mathrm{~min}$. The target gene expression levels were normalized to mouse GAPDH expression. The relative quantification gene expression of AZIN1 was determined using the $2^{-\Delta \Delta C q}$ method (25). The assay was run in triplicate for each case to allow for assessment of technical variability. To account for PCR amplification of contaminating genomic DNA, a control without reverse transcription was included. To improve the accuracy of RT-qPCR for quantification, amplifications were performed in triplicate for each RNA sample.

Western blot (WB) analysis. In the present study, an anti-AZIN1 (cat. no. orb154904; Biorbyt, Ltd.) polyclonal anti-rabbit antibodies were used to detect AZIN1 in the WB analysis. Samples were separated by SDS-PAGE on a $12.5 \%$ gel. WB was performed as described previously (26). Frozen liver tissue samples were homogenized with three volumes of $10 \mathrm{mM}$ ice cold phosphate buffer ( $\mathrm{pH} \mathrm{7.0)}$ ), containing $1 \mathrm{mM}$ ethylenediaminetetraacetic acid, $0.25 \mathrm{M}$ sucrose, $1 \mathrm{mM}$ sodium azide and $0.1 \mathrm{mM}$ phenylmethylsulfonyl fluoride. Samples were centrifuged at $20,000 \times \mathrm{g}$ for $30 \mathrm{~min}$ at $4^{\circ} \mathrm{C}$. The protein concentration was measured using a bicinchoninic acid assay (Pierce; Thermo Fisher Scientific, Inc.). The tissue lysate was obtained through denaturing electrophoresis by SDS-PAGE on a $12.5 \%$ with $60 \mathrm{mg}$ protein loaded per lane, electrotransferred to a polyvinylidene difluoride membrane, the blots was incubated with blocking buffer (1X PBS and 5\% nonfat dry milk) for $1 \mathrm{~h}$ at room temperature and then probed with primary anti-AZIN1 antibodies (1:1,000; cat. no. orb154904; Biorbyt, Ltd.) overnight at $4^{\circ} \mathrm{C}$, followed by incubation with horseradish peroxidase-conjugated secondary antibody (1:5,000; cat. no. GTX213110; GeneTex) for 1 hour at RT. To control equal loading of total protein in all lanes, blots were stained with mouse anti- $\beta$-actin antibody (1:5,000; cat. no. ab8226; Abcam). The bands were visualized using an enhanced chemiluminescence kit (GE Healthcare) according to the manufacturer's protocol. Finally, the blots were visualized using an ImageQuant ${ }^{\mathrm{TM}} \mathrm{LAS}$ 4000 system (GE Healthcare) (27).

Immunohistochemistry (IHC) analysis. AZIN1 protein expression was determined using IHC analysis of paraffin-embedded liver sections. Anti-AZIN1 IHC staining was conducted using the LAB-SA Detection System (cat. no. 85-8943; Invitrogen; Thermo Fisher Scientific, Inc.). IHC was performed as described previously (28). Paraffin tissue sections (5 $\mu \mathrm{m}$ thick) were dewaxed, treated with proteinase $\mathrm{K}$ enzyme and incubated in 3\% hydrogen peroxide for $10 \mathrm{~min}$ at room temperature to block endogenous peroxidase activity. After being washed in PBS ( $\mathrm{pH} \mathrm{7.6)} \mathrm{for} 5 \mathrm{~min}$, the sections were incubated in $0.1 \%$ Triton $\mathrm{X}$ in PBS with primary anti-AZIN1 antibody (cat. no. orb154904; 1:200 dilution; Biorbyt, Ltd.) at $4^{\circ} \mathrm{C}$ for overnight followed by staining with secondary rabbit anti-rat antibody conjugated with horseradish peroxidase $(1 \mathrm{x}$ reagent B; cat. no. 1454284A; Invitrogen; Thermo Fisher Scientific, Inc.) at room temperature for $20 \mathrm{~min}$. The immunocomplexes were visualized after treatment with 3,3'-diaminobenzidine (cat. no. 00-2014; Invitrogen; Thermo Fisher Scientific, Inc.) solution for $5 \mathrm{~min}$ at room temperature. The sections were then washed with PBS (pH 7.6) for further processing (29-31). The AZIN1 protein expression was defined using light microscopy (Leica DM 1000 LED Lab; cat. no. 10052-384; Leica Microsystems, Inc.) at a magnification of x100 or x400.

Statistical analysis. Statistically significant differences in allele/genotype frequencies of AZIN1 SNP (rs1062048) between the T2DM and control groups were determined using the $\chi^{2}$ test. Odds ratios were calculated from the genotypic frequency and allelic frequency at $95 \%$ CI for the AZIN1 SNP (rs1062048). Statistical analysis was performed using SPSS software (version 11; SPSS, Inc.). Data from three independent experiments are expressed as the mean \pm SE. Statistical comparisons between the T2DM and control groups were performed using Student's t-test. $\mathrm{P}<0.05$ was considered to indicate a statistically significant difference.

\section{Results}

Genotypic and allelic frequencies of AZIN1 gene polymorphisms in patients with T2DM and controls. Table I presents the allelic and genotypic frequencies of the rs1062048 AZIN1 gene polymorphism distribution in patients with T2DM and controls. It was observed that the T allele in the rs1062048 polymorphism was higher in the patients with T2DM $(87.6 \%$; $999 / 1,140)$ than in the controls $(85.2 \% ; 2,897 / 3,400)$. The 
Table I. Genotypic and allelic frequencies of antizyme inhibitor 1 genetic polymorphism in patients with T2DM and controls.

A, Genotype data

\begin{tabular}{lccc}
\hline Genotype & T2D (n=570), & Control $(\mathrm{n}=1,700)$, & \\
$\mathrm{n}(\%)$ & $\mathrm{n}(\%)$ & OR (95\% CI) \\
\hline CC & $12(2.1)$ & $32(1.9)$ & $1.05(0.53-2.05)$ \\
CT & $117(20.5)$ & $439(25.8)$ & $0.74(0.59-0.94)$ \\
TT & $441(77.4)$ & $1229(72.3)$ & 1.00 \\
\hline
\end{tabular}

$\mathrm{B}$, Allele frequency data

\begin{tabular}{lccc}
\hline T2DM $(\mathrm{n}=1,140)$, & Control $(\mathrm{n}=3,400)$, & \\
$\mathrm{n}(\%)$ & OR $(95 \%$ CI $)$ & $0.81(0.67-0.99)$ \\
$\mathrm{C}$ & $141(12.4)$ & $503(14.8)$ & 1.00 \\
$\mathrm{~T}$ & $999(87.6)$ & $2,897(85.2)$ & 0.042 \\
\hline
\end{tabular}

OR, odds ratio; CI, confidence interval; T2DM, type 2 diabetes mellitus.

frequencies of the TT genotype at rs1062048 differed between the control group $(72.3 \% ; 1229 / 1700)$ and patients with T2DM $(77.4 \% ; 441 / 570)$. Genotype frequencies were significantly different between T2DM and control groups $(\mathrm{P}=0.039)$. The data suggested that the T allele and TT genotype at rs1062048 SNP are risk factors for T2DM.

Downregulation of AZIN1 gene expression in liver tissues of T2DM mice. The control (+Dock $7^{\mathrm{m}} /+$ Dock $\left.7^{\mathrm{m}}\right)$ and T2DM $\left(+\right.$ Lepr $^{\mathrm{db}} /+$ Lepr $\left.^{\mathrm{db}}\right)$ mice were sacrificed at 4,16 and 32 weeks of age, and liver tissue RNA was extracted for RT-qPCR. The qPCR data in Fig. 2 shows AZIN1 gene expression in the liver tissues of mice aged 4, 16 and 32 weeks. AZIN1 gene expression in the liver tissues of mice in the T2DM group was significantly lower than that in the control group $(\mathrm{P}<0.05$; Fig. 2). These results suggested that downregulation of AZIN1 gene expression occurred in the T2DM mice.

Downregulation of AZIN1 protein expression in liver tissues of T2DM mice. Liver tissues were homogenized and $60 \mu \mathrm{g}$ protein was used in WB analysis using an anti-AZIN1 antibody and $\beta$-actin as a control. Representative blots are shown in Fig. 3A. Samples from the control mice are in lanes 1, 3 and 5 and those from the T2DM mice are in lanes 2, 4 and 6 at 4, 16 and 32 weeks, respectively. Compared with T2DM mice, no matter which stage, the AZIN1 protein expression level was always higher in the control group. In addition, the protein expression levels of AZIN1 were significantly lower in early stage (4th week) and late stage (32nd week) T2DM mouse liver samples when compared with that in control samples (Fig. 3B).

In Fig. 4, the IHC staining revealed AZIN1 protein expression in the liver tissues at 4, 16, and 32 weeks of age. AZN1 protein expression in the liver tissues of the T2DM group was lower than that in the control group (Figs. 3 and 4). The results suggested that downregulation of AZIN1 protein expression occurred in T2DM mice.

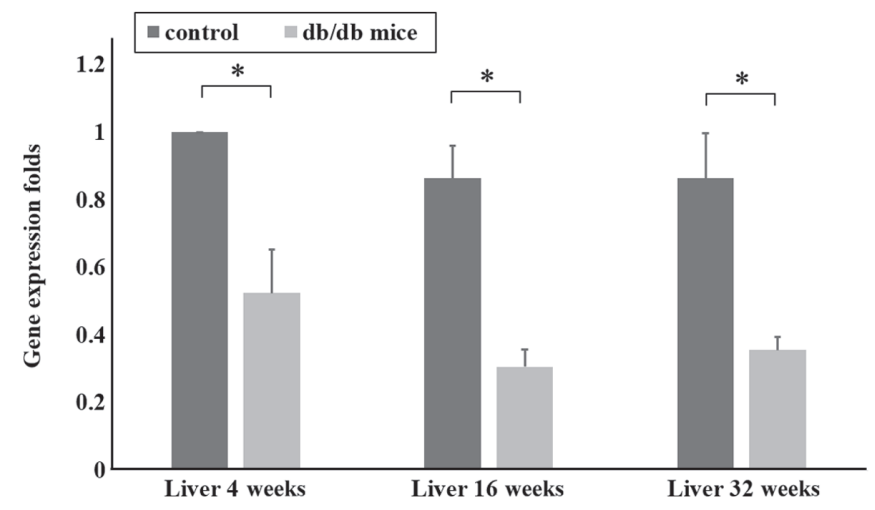

Figure 2. Reverse transcription-quantitative PCR analysis of the AZIN1 gene in the liver of control (+Dock $7^{\mathrm{m}} /+$ Dock $\left.7^{\mathrm{m}}\right)$ and type 2 diabetes mellitus $\left(+\right.$ Lepr $^{\mathrm{db}} /+$ Lepr $\left.^{\mathrm{db}}\right)$ mice at 4,16 and 32 weeks. Gene expression data of AZIN1 was calculated following normalization to GADPH. ${ }^{*} \mathrm{P}<0.05$. AZIN1, antizyme inhibitor 1 .

Association between AZIN1 rs1062048 SNP and clinical features in patients with type 2 diabetic nephropathy (T2DN). There were 246 patients with T2DM developing into T2DN in the cohort of the present study. A comparison between the clinical features of the patients with T2DN with and without the TT genotype at rs1062048 SNP is detailed in Table II. The two patient groups did not differ significantly in most of terms. However, the levels of hemoglobin A1c, blood urea nitrogen, creatinine, total calcium and triglycerides in the patients with T2DN with and without the TT genotype at rs1062048 SNP were higher than the normal values. In addition, the laboratory tests did not reveal differences between the TT genotype and non-TT genotype groups at rs1062048 SNP in the AZIN1 gene. In addition, a significantly higher level of parathyroid hormone (PTH) was observed in the TT group (202.7 \pm 176.62$)$ than in the non-TT group $(41.0 \pm 8.91 ; \mathrm{P}=0.009$; Table II). 
Table II. Association between AZIN1 rs1062048 SNP and clinical features in patients with type 2 diabetic nephropathy.

\begin{tabular}{lcccc}
\hline & \multicolumn{2}{c}{ AZIN1 genotype rs1062048 } & \\
\cline { 2 - 4 } Clinical parameters & $\begin{array}{c}\text { TT }(\mathrm{n}=189), \\
\text { mean } \pm \mathrm{SD}\end{array}$ & $\begin{array}{c}\text { Non-TT }(\mathrm{n}=57), \\
\text { mean } \pm \mathrm{SD}\end{array}$ & P-value & Normal value \\
\hline Age, years & $68.12 \pm 12.61$ & $68.28 \pm 13.0$ & 0.933 & \\
BMI, kg/m & $25.95 \pm 5.26$ & $25.38 \pm 4.84$ & 0.480 & $20-25$ \\
Hemoglobin A1c, \% & $7.22 \pm 1.64$ & $6.95 \pm 1.34$ & 0.269 & $3.8-6.0$ \\
Blood urea nitrogen, mg/dl & $38.59 \pm 26.24$ & $32.34 \pm 18.07$ & 0.066 & $7-20$ \\
Creatinine, mg/dl & $2.87 \pm 3.21$ & $2.56 \pm 2.33$ & 0.490 & $0.6-1.3$ \\
Uric acid, mg/dl & $7.16 \pm 4.31$ & $6.27 \pm 1.59$ & 0.171 & $2.3-7.6$ \\
Total calcium, mg/dl & $10.34 \pm 12.36$ & $9.20 \pm 0.65$ & 0.558 & $2.15-2.58$ \\
Phosphorus, mg/dl & $4.90 \pm 4.10$ & $4.25 \pm 0.93$ & 0.316 & $2.5-5.0$ \\
Parathyroid hormone, pg/ml & $202.76 \pm 176.62$ & $41.00 \pm 8.91$ & 0.009 & $12-65$ \\
Albumin, g/dl & $4.04 \pm 0.50$ & $4.18 \pm 0.40$ & 0.077 & $3.5-5.7$ \\
Cholesterol, mg/dl & $172.13 \pm 41.61$ & $168.49 \pm 41.65$ & 0.609 & $<200$ \\
Triglyceride, mg/dl & $181.54 \pm 162.91$ & $173.51 \pm 127.95$ & 0.762 & $<150$ \\
Low density lipoprotein-C, mg/dl & $99.32 \pm 36.84$ & $88.77 \pm 32.39$ & 0.136 & $<130$ \\
Alanine aminotransferase, IU/l & $24.64 \pm 16.09$ & $21.74 \pm 12.71$ & 0.308 & $0-41$ \\
\hline
\end{tabular}

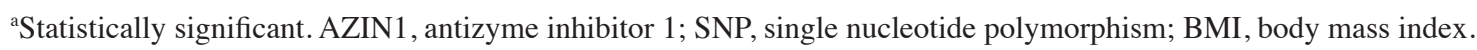

A

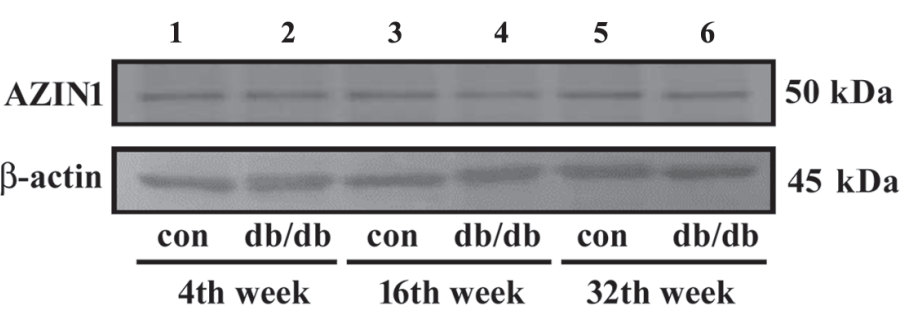

B

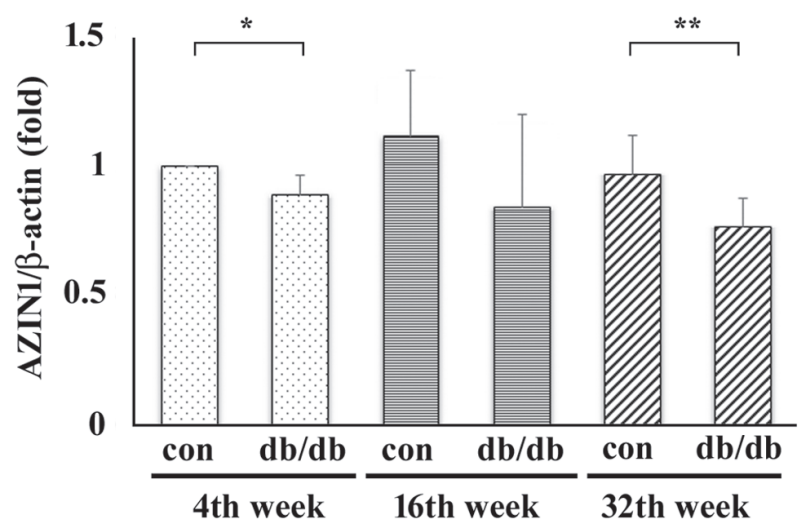

Figure 3. Hepatic AZIN1 protein expression determined by western blot analysis. (A) AZIN1 protein expression levels in the liver tissues of control (lanes 1, 3 and 5) and T2DM (lanes 2, 4 and 6) mice at 4, 16 and 32 weeks, respectively. (B) Densitometric analysis of western blotting of AZIN1 from T2DM mice at 4, 16 and 32 weeks. ${ }^{*} \mathrm{P}<0.05 ;{ }^{* *} \mathrm{P}<0.01$. AZIN1, antizyme inhibitor 1 ; con, control; db/db, mice in the T2DM group.

\section{Discussion}

According to a literature review, the present study was the first to reveal human AZIN1 gene polymorphisms in patients with T2DM. Furthermore, to the best of our knowledge, it was the first to use a time serial T2DM animal model from the early to the late stage obese mice to determine the association between
AZIN1 gene expression and protein levels by IHC staining of liver sections. The animal model exhibited the characteristics of T2DM and obesity, namely significant increases in body weight and blood glucose $(32,33)$.

Notably, the qPCR, WB and IHC assay data demonstrated downregulation of AZIN1 gene and protein expression in the T2DM group in liver tissue samples collected from mice of 


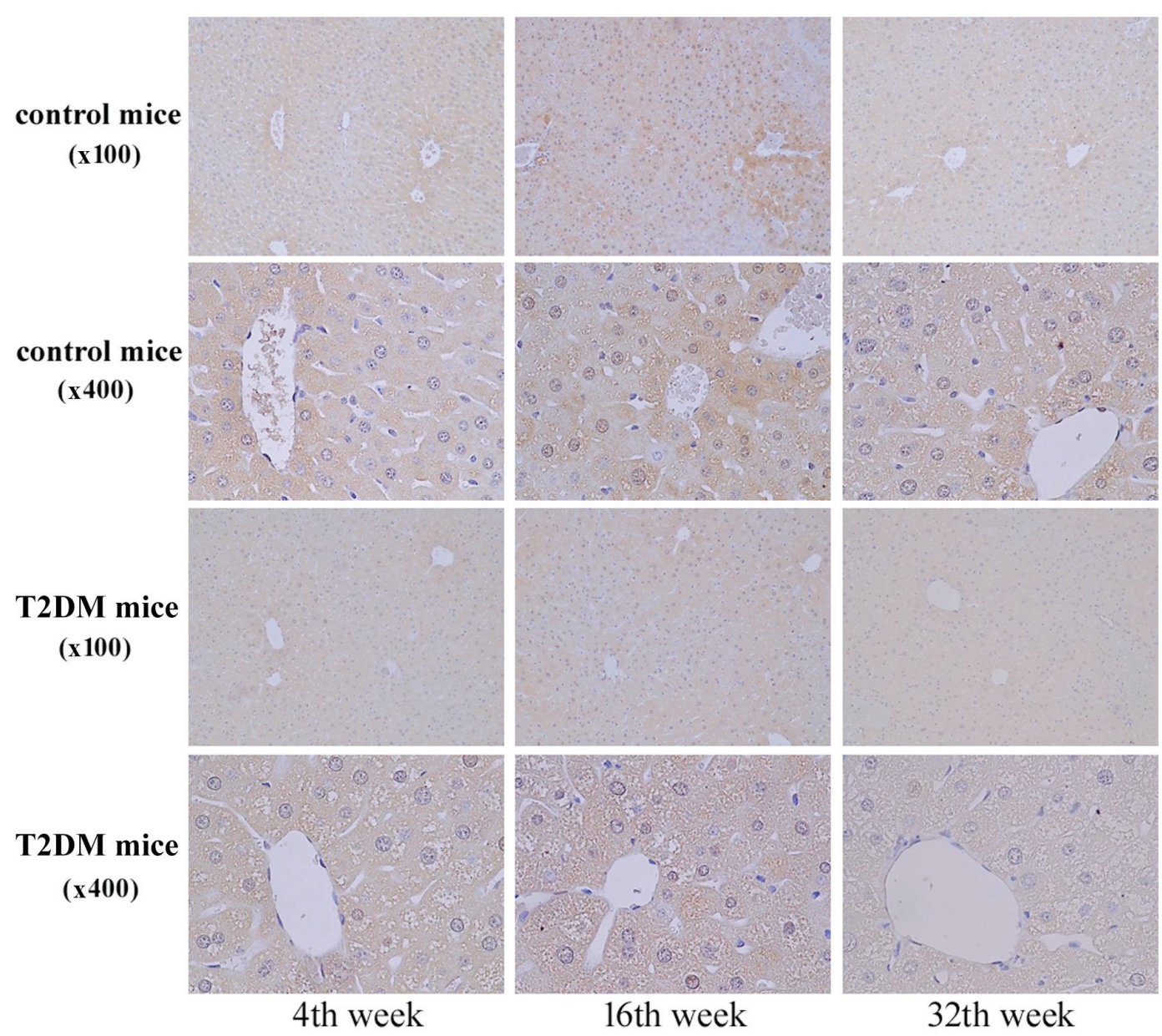

Figure 4. Representative images of the IHC analysis of antizyme inhibitor 1 expression in T2DM mice. The progression of mice model development for T2DM. Liver tissues were excised, fixed, embedded and sectioned for IHC staining. IHC staining shows representative images of positive AZIN1 expression in all liver tissue samples. Brown chromogen color indicates positive AZIN1 staining, the blue color shows the nuclear counterstaining. The magnification (x100 or x400) of the images is indicated in the figure. IHC, immunohistochemistry.

varying ages (4, 16 and 32 weeks). These findings improved the understanding of the role of AZIN1 gene expression in the pathological features of T2DM. Oka et al (18) reported that AZ1 binds to ODC, which reduces the cellular polyamine level. ASPC-1 cells are converted into $\alpha$-cells, which regulate the glucagon gene during pancreatic endocrine cell function and form an islet-like structure (18). AZIN1 effectively competes with ODC and has a higher AZ1 affinity, which restores the ODC activity of polyamine accumulation; in addition, the cellular polyamine level may serve a role in preventing diabetes (34). The animal model in the present study revealed the downregulation of AZIN1 gene and protein expression in T2DM mice. This may indirectly imply that polyamine homeostasis is impaired by a regulatory protein, such as AZ1 or AZIN1, and AZ1 can bind to ODC to reduce polyamine biosynthesis (14). Furthermore, these findings suggest that AZIN1 may serve a crucial role in the T2DM mechanism.

The present study had several limitations. First, the data indicated that the patients with T2DM exhibited a high TT genotype distribution at rs1062048 SNP in the AZIN1. However, to the best of our knowledge, no methods detecting AZIN1 activity to demonstrate the functional associations between rs1062048 SNP and the enzyme activity of AZIN1 are currently available. A second limitation was that although a time serial of the T2DM animal model was used to determine gene transcription and protein expression levels of AZIN1 by WB and IHC staining. Considering the disease/phenotype information from Jackson Laboratory (JAX stock \#000642), the phenotype findings in the present study demonstrated that compared with the control, the T2DM mice exhibited more severe fatty change during the time course of their life as they gain weight. Therefore, it is possible that liver dysfunction interfered with AZIN1 expression or its clearance. However, the level of ALT did not differ significantly between the two groups. Therefore, the liver function in all animals appeared consistent. In summary, another dedicated experimental design is required to explore the associations among AZ1/AZIN1/ODC and polyamines in human and animal models.

The data indicated that the increase of the PTH in the patients with T2DN with the TT (risk) genotype was significantly higher than that in the non-TT genotype patients. Recently, Mary et al (35) reported that higher PTH prevalence is associated with increased below-the-knee arterial calcification in patients with T2DM. Therefore, the present study suggested that the patients with T2DN with the TT (risk) genotype were at risk of increased calcification of the inferior knee artery due to a high prevalence of PTH. 
In conclusion, the present study explored the downregulation of AZIN1 gene and protein expression in T2DM mice, and demonstrated the association between AZIN1 and T2DM. To the best of our knowledge, the present study was the first to use a time serial animal model to investigate the association between rodent AZIN1 gene expression and the progression of T2DM disease from the gene expression to the phenotype level. Further research is required to understand the mechanisms of AZIN1 at the gene and protein levels, and its association with pancreatic endocrine cells in patients with T2DM.

\section{Acknowledgements}

Not applicable.

\section{Funding}

The present study was supported by China Medical University Hospital in Taiwan (grant nos. DMR-104-082 and DMR-106-116) and by two funds from the National Health Research Institutes (grant nos. 07A1-MGSP08-037 and TCVGH-NHRI10705).

\section{Availability of data and materials}

The datasets used and/or analyzed during the present study available from the corresponding author on reasonable request.

\section{Authors' contributions}

SYC, CHC, YHW and FJT conceived and designed the study. SYC, CHC, YHW, FJT, SFT and TMY analyzed and interpreted the data. SYC, CHC, YHW and FJT participated in the drafting of the manuscript. SYC, CHC, YHW, FJT, SFT and TMY critically revised the manuscript for important intellectual content. All authors read and approved the final manuscript.

\section{Ethics approval and consent to participate}

The animal experiments were approved by the Institutional Animal Care and Use Committee of China Medical University (IACUC permit no. 2016-221). The human experiments were approved by the Ethics Committee/Institutional Review Board of China Medical University Hospital (approval no. CMUH103-REC2-071). All participants provided written informed consent.

\section{Patient consent for publication}

Not applicable.

\section{Competing interests}

The authors declare that they have no competing interests.

\section{References}

1. World Health Organization (WHO): Global Report on Diabetes. WHO, Geneva, 2016.

2. International Diabetes Federation (IDF): IDF Diabetes Atlas, 8th Edition. International Diabetes Federation, Brussels, 2017.
3. Kodama K, Tojjar D, Yamada S, Toda K, Patel CJ and Butte AJ: Ethnic differences in the relationship between insulin sensitivity and insulin response: A systematic review and meta-analysis. Diabetes Care 36: 1789-1796, 2013.

4. Ma RC and Chan JC: Type 2 diabetes in East Asians: Similarities and differences with populations in Europe and the United States. Ann N Y Acad Sci 1281: 64-91, 2013.

5. Tatsumi Y, Morimoto A, Miyamatsu N, Noda M, Ohno Y and Deura K: Effect of body mass index on insulin secretion or sensitivity and diabetes. Am J Prev Med 48: 128-135, 2015.

6. Chung HF, Al Mamun A, Huang MC, Long KZ, Huang YF, Shin SJ, Hwang SJ and Hsu CC: Obesity, weight change, and chronic kidney disease in patients with type 2 diabetes mellitus: A longitudinal study in Taiwan. J Diabetes 9: 983-993, 2017.

7. Groop LC and Tuomi T: Non-insulin-dependent diabetes mellitus-a collision between thrifty genes and an affluent society. Ann Med 29: 37-53, 1997.

8. Tanda R, Anderson SE and Kamboj MK: Pediatric type 2 diabetes: Prevention and treatment. In: The Handbook of Life Course Health Development. Halfon N, Forrest CB, Lerner RM, Faustman EM (eds). Springer, New York, NY, pp197-236, 2017.

9. Lu CC, Chen YT, Chen SY, Hsu YM, Lin CC, Tsao JW, Juan YN, Yang JS and Tsai FJ: Hematopoietically expressed homeobox gene is associated with type 2 diabetes in KK $\mathrm{Cg}-\mathrm{A}^{\mathrm{y}} / \mathrm{J}$ mice and a Taiwanese Han Chinese population. Exp Ther Med 16: 185-191, 2018.

10. Chen SY, Hsu YM, Lin YJ, Huang YC, Chen CJ, Lin WD, Liao WL, Chen YT, Lin WY, Liu YH, et al: Current concepts regarding developmental mechanisms in diabetic retinopathy in Taiwan. Biomedicine (Taipei) 6: 7, 2016.

11. Yang JS, Lu CC, Kuo SC, Hsu YM, Tsai SC, Chen SY, Chen YT, Lin YJ, Huang YC, Chen CJ, et al: Autophagy and its link to type II diabetes mellitus. Biomedicine (Taipei) 7: 8, 2017.

12. Prasad RB and Groop L: Genetics of type 2 diabetes-pitfalls and possibilities. Genes (Basel) 6: 87-123, 2015.

13. Park MH and Igarashi K: Polyamines and their metabolites as diagnostic markers of human diseases. Biomol Ther (Seoul) 21: 1-9, 2013.

14. Wu HY, Chen SF, Hsieh JY, Chou F, Wang YH, Lin WT, Lee PY, Yu YJ, Lin LY, Lin TS, et al: Structural basis of antizyme-mediated regulation of polyamine homeostasis. Proc Natl Acad Sci USA 112: 11229-11234, 2015.

15. Matsufuji S, Matsufuji T, Miyazaki Y, Murakami Y, Atkins JF, Gesteland RF and Hayashi S. Autoregulatory frameshifting in decoding mammalian ornithine decarboxylase antizyme. Cell 80: 51-60, 1995.

16. Rom E and Kahana C: Polyamines regulate the expression of ornithine decarboxylase antizyme in vitro by inducing ribosomal frame-shifting. Proc Natl Acad Sci USA 91: 3959-3963, 1994.

17. Qiu S, Liu J and Xing F: Antizyme inhibitor 1: A potential carcinogenic molecule. Cancer Sci 108: 163-169, 2017.

18. Oka T, Ohtani M and Suzuki J: Identification of novel molecules regulating differentiation and hormone secretion and clarification of their functional mechanisms in pancreatic endocrine cells. Yakugaku Zasshi 130: 377-388, 2010 (In Japanese).

19. American Diabetes Association: Diagnosis and classification of diabetes mellitus. Diabetes Care 36 (Suppl 1): S67-S74, 2013.

20. Ibrahim DM: Reduce, Refine, Replace: The Failure of the Three R's and the Future of Animal Experimentation. University of Chicago Legal Forum: Vol. 2006, Article 7, 2006.

21. Mandel MA and Mahmoud AA: Impairment of cell-mediated immunity in mutation diabetic mice (db/db). J Immunol 120: 1375-1377, 1978.

22. Didovyk A, Tonooka T, Tsimring L and Hasty J: Rapid and scalable preparation of bacterial lysates for cell-free gene expression. ACS Synth Biol 6: 2198-2208, 2017.

23. Chen YT, Liao JW, Tsai YC and Tsai FJ: Inhibition of DNA methyltransferase 1 increases nuclear receptor subfamily 4 group A member 1 expression and decreases blood glucose in type 2 diabetes. Oncotarget 7: 39162-39170, 2016.

24. Bleda S, de Haro J, Varela C, Ferruelo A and Acin F: Elevated levels of triglycerides and vldl-cholesterol provoke activation of nlrp1 inflammasome in endothelial cells. Int J Cardiol 220: 52-55, 2016. 
25. Livak KJ and Schmittgen TD: Analysis of relative gene expression data using real-time quantitative PCR and the 2(-Delta Delta C(T)) method Methods 25: 402-408, 2001.

26. Wu KM, Hsu YM, Ying MC, Tsai FJ, Tsai CH, Chung JG, Yang JS, Tang CH, Cheng LY, Su PH, et al: High-density lipoprotein ameliorates palmitic acid-induced lipotoxicity and oxidative dysfunction in H9c2 cardiomyoblast cells via ROS suppression. Nutr Metab (Lond) 16: 36, 2019.

27. Huang CY, Chen SY, Tsai HC, Hsu HC and Tang CH: Thrombin induces epidermal growth factor receptor transactivation and CCL2 expression in human osteoblasts. Arthritis Rheum 64: 3344-3354, 2012.

28. Chen SY,Lin JR, Darbha R, Lin P, Liu TY and Chen YM: Glycine $\mathrm{N}$-methyltransferase tumor susceptibility gene in the benzo(a) pyrene-detoxification pathway. Cancer Res 64: 3617-3623, 2004.

29. Steneberg P, Bernardo L, Edfalk S, Lundberg L, Backlund F Ostenson CG and Edlund H: The type 2 diabetes-associated gene ide is required for insulin secretion and suppression of $\alpha$-synuclein levels in $\beta$-cells. Diabetes 62: 2004-2014, 2013.

30. Sekiguchi K, Kurabayashi M, Oyama Y, Aihara Y, Tanaka T, Sakamoto H, Hoshino Y, Kanda T, Yokoyama T, Shimomura Y, et al: Homeobox protein Hex induces SMemb/nonmuscle myosin heavy chain-B gene expression through the cAMP-responsive element. Circ Res 88: 52-58, 2001.
31. Cotsapas C, Prokunina-Olsson L, Welch C, Saxena R, Weaver C, Usher N, Guiducci C, Bonakdar S, Turner N, LaCroix B and Hall JL: Expression analysis of loci associated with type 2 diabetes in human tissues. Diabetologia 53: 2334-2339, 2010.

32. Chen H, Charlat O, Tartaglia LA, Woolf EA, Weng X, Ellis SJ, Lakey ND, Culpepper J, Moore KJ, Breitbart RE, et al: Evidence that the diabetes gene encodes the leptin receptor: Identification of a mutation in the leptin receptor gene in $\mathrm{db} / \mathrm{db}$ mice. Cell 84: 491-495, 1996.

33. Hummel KP, Dickie MM and Coleman DL: Diabetes, a new mutation in the mouse. Science 153: 1127-1128, 1966.

34. Palanimurugan R, Scheel H, Hofmann K and Dohmen RJ: Polyamines regulate their synthesis by inducing expression and blocking degradation of ODC antizyme. EMBO J 23: 4857-4867, 2004.

35. Mary A, Hartemann A, Brazier M, Aubert CE, Kemel S, Salem JE, Cluzel P, Liabeuf S, Massy Z, Mentaverri R, et al: Higher parathyroid hormone levels are associated with increased below-the-knee arterial calcification in type 2 diabetes. Diabetes Metab 44: 305-308, 2018 